Rademakers, J., Delnoij, D., Boer, D. de. Structure, process or outcome: which contributes most to patients' overall assessment of healthcare quality? BMJ Quality and Safety: 2011, 20(4), 326-331

\begin{tabular}{|l|l|}
\hline Postprint Version & 1.0 \\
\hline Journal website & $\underline{\mathrm{http}: / / q u a l i t y s a f e t y . b m j . c o m / c o n t e n t / e a r l y / 2011 / 02 / 21 / b m j q s .2010 .042358 . a b s t r}$ \\
\hline Pubmed link & $\underline{\underline{\mathrm{act}}}$ \\
\hline DOI & $\underline{\mathrm{htp}: / / \text { www.ncbi.nlm.nih.gov/pubmed/21339310 }}$ \\
\hline
\end{tabular}

This is a NIVEL certified Post Print, more info at http://www.nivel.eu

\title{
Structure, process or outcome: which contributes most to patients' overall assessment of healthcare quality?
}

\author{
JANY RADEMAKERS $_{1}$, DIANA DELNOIJ 2 , DOLF DE BOER ${ }_{1,2}$
}

${ }_{1}$ Netherlands Institute for Health Services Research, Utrecht, The Netherlands

${ }^{2}$ Centre for Consumer Experiences in Healthcare, Utrecht, The Netherlands

\begin{abstract}
Research questions: The paper explores which type of quality aspects (structure, process, outcome) most strongly determines patients' overall assessment of healthcare, and whether there is a variation between different types of patient groups in this respect.

Methods: Secondary analyses were undertaken on survey data from patients who underwent hip or knee surgery, cataract surgery, patients suffering from varicose veins, spinal disc herniation or rheumatoid arthritis. In these analyses, the patient-given global rating served as the dependent variable, and experiences regarding structure (waiting times, continuity of care), process (doctor-patient communication and information) and outcome aspects (improvement or worsening of symptoms) served as independent variables.

Results: Experiences regarding process aspects explained most of the variance in the global rating (16.4-23.3\%), followed by structure aspects (8.1-21.0\%). Experiences regarding outcome did not explain much variance in the global rating in any of the patient groups (5.313.5\%). The patient groups did not differ with respect to the type of quality aspects that most predicted the overall assessment.

Discussion: Improving process and structure aspects of healthcare is most likely to increase patients' overall evaluation of the quality of care as expressed in a global rating. A more sophisticated method of patient reported outcome measurement, with pre- and post-treatment questionnaires and the inclusion of quality-of-life criteria, might lead to higher associations between outcome and the overall evaluation of the received care.
\end{abstract}

\section{INTRODUCTION}

Since many Western countries have introduced a market-oriented healthcare system, assessing quality of care from the patients' perspective has become increasingly important. In these systems, the role of the patient has changed from being a passive recipient of care to acting as active consumers. In order for patients to make informed choices, they need comparable information about the performance of healthcare providers regarding quality and safety. Transparency has become a professional value and a tool in creating more open and balanced relationships between service providers and the public.1 However, the definition of quality indicators and measures is not value-free, and different stakeholders tend to perceive quality of care differently.2 3 Patients offer a complementary perspective to that of providers and policy makers and should therefore be involved in the process of developing quality criteria, indicators and measuring 
Rademakers, J., Delnoij, D., Boer, D. de. Structure, process or outcome: which contributes most to patients' overall assessment of healthcare quality? BMJ Quality and Safety: 2011, 20(4), 326-331

instruments.4 They may add relevant topics and set priorities which enhances the validity of the instruments, thus preventing that quality of care is 'mislabelled or mismeasured' from the patient's point of view.5

In his well-known model of quality of healthcare, the physician Donabedian distinguished three components: structure, process and outcome.6 He defined structure as the environment in which healthcare is provided, process as the method by which healthcare is provided and outcome as the consequence of the healthcare provided. Since quality of care is theoretically based on aspects from all three components, they should all be included in questionnaires and interview protocols on patient satisfaction or patient experiences. In many instruments designed to measure patients' experiences with quality of healthcare, such as the American Consumer Assessment of Health Plans and Providers (CAHPS) and the Dutch Consumer Quality-index (CQ-index), elements of all three components have indeed been included.5 78 In most patient experience questionnaires, apart from individual quality aspects, a global rating or overall assessment on the quality of healthcare received is obtained as well.9-11 In both the CAHPS and the CQindex questionnaires, global ratings are measured on a scale from 0 to 10. In The Netherlands, this rating scale is similar to the school grading system and therefore very well known to patients. These ratings are often used as summary information regarding the quality of care, and presented in public communications, but it is not quite clear what they actually represent. Several authors have found high correlations between process aspects and the overall assessment of healthcare.9 10 In general, various studies have shown the importance of process aspects (ie, providers affective style, communication, information) for patients.11-14 A recent study showed that global ratings are marginally dependent on demographics, and that experiences regarding quality aspects that patients regard as important are somewhat better predictors of the global rating compared with experiences on aspects that patients considered less important. Since priorities varied for specific patient groups, the global rating in specific patient experience questionnaires reflected different quality aspects accordingly.15

The present paper will explore the extent to which structure, process and outcome aspects are reflected in the global ratings patients administer, and whether, similar to the study by De Boer et al,15 there are any differences in this respect between patient groups. One hypothesis might be that for patients who suffer from a chronic disease from which total recovery is unlikely, process aspects would be more important in determining the overall quality of care, whereas for patients who undergo a single surgical procedure, structure and especially outcome aspects may be stronger determinants of the global rating. This study is particularly relevant for professionals and managers in healthcare because it elucidates whether they have to focus on structure, process or outcome aspects to increase patient satisfaction, measured in terms of global ratings. The following research questions will be addressed:

Which type of quality aspect (structure, process, outcome) most strongly determines patients' overall assessment of healthcare?

Is there any variation between different types of patient groups in this respect?

\section{METHODS}

\section{Participants}

Survey data were used from patients who underwent hip or knee surgery ( $\mathrm{n}=1686$, response $=75.0 \%$ ) or cataract surgery $(n=4635$, response $=71.6 \%)$, and patients suffering from varicose veins $(n=1138$, response $=63 \%$ ), spinal disc herniation $(n=615$, response $=51.6 \%)$ or rheumatoid arthritis $(n=2757$, response $=72.7 \%$ ). Data on the demographic characteristics age, self-rated health, education and sex are presented in table 1.

\section{[TABLE 1]}

All data were collected in The Netherlands. Patients were identified through insurance companies and/or hospitals, and approached by mail using a procedure known as the Dillman method, 16 which includes up to three reminders if necessary. Importantly, the patients approached were derived from a number of healthcare providers, thereby ensuring that our results do not reflect an isolated case. The questionnaires were sent within 12 months after treatment or surgery. These five groups were selected because of the wide variety of patients they represent, from patients with a chronic illness (rheumatoid arthritis17) to patients who underwent (predominantly) planned one-time surgery (hip/knee18 or cataract surgery).19 Patients with 
Rademakers, J., Delnoij, D., Boer, D. de. Structure, process or outcome: which contributes most to patients' overall assessment of healthcare quality? BMJ Quality and Safety: 2011, 20(4), 326-331

varicose veins or spinal disc herniation constitute a separate group, since their treatment is aimed at recovery, but many different treatment options can be part of the process.

\section{Measurement of experiences regarding structure, process and outcome}

CQ-index surveys are tailored towards various patient groups. Obviously aspects of care, such as communication with the healthcare provider, are similar for most or all patient groups and, consequently, occur in a generic format in most surveys. Other aspects, such as the desired outcome, vary greatly between patient groups and require items specifically designed for each group. Answering categories for CQ-index items typically focus on the frequency with which quality criteria were met on a scale from 1 to 4 (ie, never, sometimes, usually, always) and the extent to which performance on quality criteria has raised problems on a scale from 1 to 3 (ie, big problem, small problem, no problem). Other answering categories (such as yes or no) are employed where categories regarding magnitude of problems or frequency of meeting quality criteria are not appropriate. For the patient groups at issue, the CQ-index surveys were inspected for elements regarding process, structure and outcome. As measures of process, three items were selected that focused on doctor-patient communication: doctor listens carefully, doctor explains clearly, and doctor includes patient in decision-making. The items were virtually identical for all surveys. For measures of structure, we focused on waiting times and continuity of care, as these were represented in each survey. For waiting times, one item concerning problems with waiting times following referral was selected, which was similar across the surveys at issue. The second item entailed the actual waiting time in case of hip or knee surgery and spinal disc herniation, and experiences with prompt responses when contacting the healthcare provider for cataract surgery, varicose veins and rheumatoid arthritis. For continuity of care, one item was selected concerning the consistency of patient information or the extent to which information was successfully transferred between healthcare providers. The second item concerned the extend to which various healthcare professionals synchronised their treatments. For hip or knee surgery, such items were not available, so we selected an item concerning the presence of a case manager and an item concerning the availability of a joint care programme instead. Further, items assessing the extent to which physical functioning was improved as compared with the start of treatment were selected. For all surveys, the answering categories were worse-similar-better as compared with the start of treatment. For the survey for cataract surgery, these items covered issues such as being able to see things from a close distance or far away, being able to cope with bright lights, being able to drive, etc. For the survey on hip or knee surgery, these items entailed issues such as stair climbing, pain, standing, walking, etc. In the case of varicose veins, these items covered issues such as feelings of fatigue in the legs, pain, standing, physical appearance, etc. For spinal disc herniation, these items covered issues such as stairclimbing, standing up, walking, back pain, mobility, etc. For each survey, these items were summarised by the construction of three variables: a variable containing the number of elements that deteriorated, the number of elements that stayed similar and the number of elements that improved. Finally, the global rating of care (measured on a scale from 0 to 10) was selected for each patient group.

\section{Data analyses}

Data analyses were focused on the proportion of variance in global rating that may be explained by experiences regarding process-, structure- or outcome-related aspects. To that extent, linear regression analyses were performed in which the global rating served as the dependent variable, and experiences regarding structure, process or outcome served as independent variables. We employed separate models for structure, process and outcome to circumvent possible multicollinearity between structure, process and outcome variables. Further, we focused on the variance explained, rather than coefficients of independent variables, as the variance explained is not influenced by possible multicollinearity between independent variables within the separate models for structure, process or outcome variables. We controlled for the demographic variable age, education and self-rated health. Analyses were performed using the statistical package SPSS 14.0.

\section{RESULTS}

Table 2 lists the results of the linear regression analyses.

\section{[TABLE 2]}


Rademakers, J., Delnoij, D., Boer, D. de. Structure, process or outcome: which contributes most to patients' overall assessment of healthcare quality? BMJ Quality and Safety: 2011, 20(4), 326-331

For all patient groups, experiences regarding process aspects (communication, information, patient involvement in decision-making) explained most of the variance in the global rating (16.4-23.3\%), followed by structure aspects (8.1-21.0\%). Especially in the patient groups that underwent hip/knee or cataract surgery and the patients with a spinal disc herniation, structure aspects (waiting times, prompt responses and continuity of care) were important determinants of the global rating (15.1-20.1\%), Further, experiences regarding outcome (improvement of physical functioning) did not explain much variance in the global rating in any of the patient groups (5.3-13.5\%). There were no differences between the patient groups with respect to the type of quality aspects that most predicted the overall assessment.

\section{DISCUSSION}

In this study among five different patient groups, experiences regarding process aspects of care explained most of the variance in the overall assessment, measured in terms of a global rating. Experiences with structure aspects were the second most important predictors. Experiences regarding outcome of the treatment did not explain much variance in the global rating. The patient groups did not differ with respect to the type of quality aspects that most predicted the overall assessment: for all groups, process aspects proved to be the most important predictors, then structure aspects and last outcome.

Improving process and structure aspects of healthcare is most likely to increase patients' overall evaluation of the quality of care. In the present study, process was defined as doctor-patient communication and information: being listened to, having received clear explanations and having been involved in shared decision-making. In the questionnaires included in this study, the questions on these three process aspects were virtually identical, which enhanced comparability. The relative importance of communication and information is congruent with previous international research9-14 and seems to be applicable to other health professionals (nurses, physical therapists) as well. The relative importance of structural aspects (in this study defined as waiting times and continuity of care) for patients' overall assessment has not been demonstrated in earlier studies. In general, structure is regarded as the least researched topic of Donabedian's triad in the quality-improvement literature.20 21 Structure aspects and how they impact patients' overall assessment of care require further examination. In countries where the access to healthcare services is more difficult and variable than in The Netherlands, the impact of an aspect such as waiting time might even be greater. However, other structural aspects which are less visible to patients, such as the availability of a quality policy, may have a lesser impact.

Judging from the present data, improvement efforts focused on outcome should not be evaluated by changes in the patient-given global rating, as associations between patient experiences regarding outcome and the global rating are modest. This does not necessarily mean that the outcome of their treatment is not important to patients. Since improvement in functional status or relief of symptoms, in any respect, is generally a treatment effect, this kind of outcome might not be discriminatory with respect to the overall assessment. In patient experience questionnaires such as the CQ index, outcome is only measured retrospectively and from a narrow conceptual perspective. Possibly a more sophisticated method of patient reported outcome measurement, with pre- and post-treatment questionnaires and the inclusion of quality-oflife criteria, might lead to higher associations between outcome and the overall evaluation of the received care. In several studies or conceptual models, patient satisfaction itself is considered a treatment outcome. 3 Though positive patient experiences and evaluations should certainly be the ambition of healthcare professionals, satisfaction in itself is a multidimensional and subjective concept which is neither valid nor specific enough to measure quality of care from the patients' perspective. This study shows that patient satisfaction, as measured in a global rating, is associated with specific process and structure aspects. This information can be used by professionals and managers in healthcare to further improve their services. In some countries, data from patient experience surveys have already been used as input for quality improvement projects.22

A limitation of our study is that not all questionnaires included the exact same measures for (especially) structure and outcome aspects. Different items had to be used for different patient groups when defining waiting times and continuity of care. Though this does not present a major problem for the purpose of the analysis conducted here, the specific and sometimes narrow definitions used may warrant caution when generalising these findings to structure or outcome aspects in general. 
Rademakers, J., Delnoij, D., Boer, D. de. Structure, process or outcome: which contributes most to patients' overall assessment of healthcare quality? BMJ Quality and Safety: 2011, 20(4), 326-331

\section{REFERENCES}

1. Berenson R, Cassel CK. Consumer-driven health care may not what patients needdCaveat emptor. JAMA 2009;3:321e3.

2. Donabedian A. Advantages and limitations of explicit criteria for assessing the quality of health care. Millbank Q 1981;59:99e106.

3. Campbell SM, Roland MO, Buetow SA. Defining quality of care. Soc Sci Med 2000;51:1611e25.

4. Black N, Jenkinson C. Measuring patients' experiences and outcomes. BMJ 2009;339:b2495.

5. Feinstein AR. Is 'quality of care' being mislabeled or mismeasured? Am J Med 2002;112:472e8.

6. Donabedian A. Explorations in Quality Assessment and Monitoring (Vol. 1): the Definition of Quality and Approaches to Its Assessment. Ann Arbor, MI: Health Administration Press, 1980.

7. http://www.ahrq.gov/qual/cahps.htm.

8. Rademakers J. Measuring patients' experiences with quality of health-care; lessons from the Consumer Quality-index. Eur J Public Health 2008;18(Suppl 1):58.

9. Arah OA, ten Asbroek AH, Delnoij DM, et al. Psychometric properties of the Dutch version of the Hospital-level Consumer Assessment of Health Plans Survey instrument. Health Serv Res 2006;41:284e301.

10. Otani K. Enrollees' global rating process of health care with the national CAHPS Benchmarking Database. Health Care Manag Rev 2006;31:205e12.

11. Hibbard JH, Jewett JJ. Will quality report cards help consumers? Health Aff 1997;16:218e28.

12. Bensing JM. Doctorepatient communication and the quality of care.

Soc Sci Med 1991;32:1301e10.

13. Krupat E, Fancey M, Cleary PD. Information and its impact on satisfaction among surgical patients. Soc Sci Med 2000;5:1817e25.

14. Ong L, de Haes J, Hoos A, et al. Doctorepatient communication: a review of the literature. Soc Sci Med 1995;40:903e18.

15. de Boer D, Delnoij D, Rademakers J. Do patient experiences on priority aspects of health care predict their global rating of quality of care? A study in five patient groups. Health Expect 2010;13:285e97.

16. Dillman DA. Mail and Internet Surveys: The Tailored Design Method.

New York: Wiley, 2000.

17. Zuidgeest M, Sixma H, Rademakers J. Measuring patients' experiences with rheumatic care: the Consumer Quality Index Rheumatoid Arthritis. Rheumatol Int 2009;30:159e67.

18. Stubbe J, Gelsema T, Delnoij DMJ. The Consumer Quality Index Hip Knee Questionnaire measuring patients' experience with quality of care after a total hip or knee arthroplasty. BMC Health Serv Res 2007;7:60.

19. Stubbe J, Brouwer W, Delnoij DMJ. Patient's experiences with quality of hospital care: the Dutch Consumer Quality Index Cataract Questionnaire. BMC Ophtalmol 2007;7:14.

20. Meyer GS, Masagli MO. The forgotten component of the quality triad, can we still learn something from 'structure'? Jt Comm J Qual Improv 2001;27:484e93.

21. Glickman SW, Baggett KA, Krubert CG, et al. Promoting quality: the health-care organization from a management perspective. Int J Qual Health Care 2007;19:341e8.

22. Davies E, Shaller D, Edgman-Levitan S, et al. Evaluating the use of a modified CAHPS survey to support improvements in patientcentred care: lessons from a quality improvement collaborative. Health Expect 2008;11:160e76. 
Rademakers, J., Delnoij, D., Boer, D. de. Structure, process or outcome: which contributes most to patients' overall assessment of healthcare quality? BMJ Quality and Safety: 2011, 20(4), 326-331

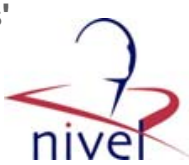

[TABLES]

\begin{tabular}{|c|c|c|c|c|c|c|c|c|c|}
\hline & \multicolumn{9}{|l|}{ Education } \\
\hline & 1 & 2 & 3 & 4 & 5 & 6 & 7 & 8 & Missing \\
\hline Hip or knee surgery & $20(1.9 \%)$ & $436(25.9 \%)$ & $538(31.9 \%)$ & $285(16.9 \%)$ & $126(7.5 \%)$ & $68(4.0 \%)$ & $69(4.1 \%)$ & $23(1.4 \%)$ & $121(7.2 \%)$ \\
\hline Cataract surgery & $90(1.9 \%)$ & $1390(30.0 \%)$ & $1259(27.2 \%)$ & $808(17.4 \%)$ & $269(5.8 \%)$ & $163(3.5 \%$ & $262(5.7 \%)$ & $82(1.8 \%)$ & $312(6.7 \%)$ \\
\hline Varicose veins & $11(1.5 \%)$ & $22(3.1 \%)$ & $130(18.3 \%)$ & $161(22.6 \%)$ & $142(20.0 \%)$ & $61(8.6 \%)$ & $106(14.9 \%)$ & $39(5.5 \%)$ & $39(5.5 \%)$ \\
\hline Rheumatoid arthritis & $116(4.2 \%)$ & $394(14.3 \%)$ & $760(27.6 \%)$ & $581(21.1 \%)$ & $343(12.4 \%)$ & $145(5.3 \%$ & $252(9.1 \%)$ & $57(2.1 \%)$ & $109(4.0 \%)$ \\
\hline \multirow[t]{3}{*}{ Spinal disc herniation } & $8(1.3 \%)$ & $41(6.7 \%)$ & $141(22.9 \%)$ & $114(18.5 \%)$ & $124(20.2 \%)$ & $35(5.7 \%)$ & $102(16.6 \%)$ & $36(5.9 \%)$ & $14(2.3 \%)$ \\
\hline & \multicolumn{9}{|l|}{ Age } \\
\hline & $18-24$ & $25-34$ & $35-44$ & $45-54$ & \multicolumn{2}{|l|}{$55-64$} & $65-74$ & $75-79$ & Missing \\
\hline Hip or knee surgery & $1(0.1 \%)$ & $8(0.5 \%)$ & $22(1.3 \%)$ & $85(5.0 \%)$ & \multicolumn{2}{|c|}{$358(21.2 \%)$} & $(38.7 \%)$ & $544(32.3 \%)$ & $15(0.9 \%)$ \\
\hline Cataract surgery & $2(0.0 \%)$ & $15(0.3 \%)$ & $23(0.5 \%)$ & $148(3.2 \%)$ & \multicolumn{2}{|c|}{$530(11.4 \%)$} & $2(32.0 \%)$ & $2338(50.4 \%)$ & $97(2.1 \%)$ \\
\hline Varicose veins & $6(0.8 \%)$ & $45(6.3 \%)$ & $139(19.5 \%)$ & \multirow{2}{*}{$\begin{array}{l}163(22.9 \%) \\
388(14.1 \%)\end{array}$} & \multicolumn{2}{|c|}{$212(29.8 \%)$} & $(16.7 \%)$ & $20(2.8 \%)$ & $7(1.0 \%)$ \\
\hline Rheumatoid arthritis & $10(0.4 \%)$ & $69(2.5 \%)$ & $162(5.9 \%)$ & & \multicolumn{2}{|c|}{$745(27.0 \%)$} & $(28.1 \%)$ & $591(21.4 \%)$ & $18(0.7 \%)$ \\
\hline \multirow[t]{3}{*}{ Spinal disc herniation } & $10(1.6 \%)$ & $33(5.4 \%)$ & $127(20.7 \%)$ & $181(29.4 \%)$ & $108(17$ & $6 \%)$ & $(16.6 \%)$ & $49(8.0 \%)$ & $5(0.8 \%)$ \\
\hline & \multicolumn{6}{|c|}{ Self-rated health } & \multicolumn{3}{|l|}{ Gender } \\
\hline & Poor & Fair & Good & Very good & Excellent & Missing & Female & Male & Missing \\
\hline Hip or knee surgery & $14(0.8 \%)$ & $214(12.7 \%)$ & $847(50.2 \%)$ & $280(16.6 \%)$ & $285(16.9 \%)$ & $46(2.7 \%)$ & $1200(71.2 \%)$ & $471(27.9 \%)$ & $15(0.9 \%)$ \\
\hline Cataract surgery & $310(6.7 \%)$ & $510(11.0 \%)$ & $2286(49.2 \%)$ & $1245(26.9 \%)$ & $110(2.4 \%)$ & $174(3.8 \%)$ & $2870(61.9 \%)$ & $1685(36.4 \%)$ & $80(1.7 \%)$ \\
\hline Varicose veins & $3(0.4 \%)$ & $66(9.3 \%)$ & $447(62.9 \%)$ & $137(19.3 \%)$ & $47(6.6 \%)$ & $11(1.5 \%)$ & $546(76.8 \%)$ & $119(16.7 \%)$ & $48(6.5 \%)$ \\
\hline Rheumatoid arthritis & $113(4.1 \%)$ & $1282(46.5 \%)$ & $1194(43.3 \%)$ & $78(2.8 \%)$ & $26(0.9 \%)$ & $64(2.3 \%)$ & $1906(69.1 \%)$ & $815(29.6 \%)$ & $36(1.3 \%)$ \\
\hline Spinal disc herniation & $22(3.6 \%)$ & $162(26.3 \%)$ & $297(48.3 \%)$ & $87(14.1 \%)$ & $35(5.7 \%)$ & $12(2.0 \%)$ & $305(49.6 \%)$ & $304(49.4 \%)$ & $6.0(1.0 \%)$ \\
\hline
\end{tabular}

Table 2 Linear regression analyses with the global rating as the dependent variable and experiences regarding structure, process or outcome as independent variables

\begin{tabular}{llllll}
\hline & Model & Adjusted $\mathbf{R}^{2}$ & df1 & df2 & Significance (p) \\
\hline Hip or knee surgery & dc & 0.065 & 3 & 1682 & $<0.01$ \\
& dc. process & 0.201 & 3 & 1679 & $<0.01$ \\
& dc. structure & 0.151 & 4 & 1678 & $<0.01$ \\
Cataract surgery & dc. outcome & 0.095 & 3 & 1679 & $<0.01$ \\
& dc & 0.025 & 3 & 4631 & $<0.01$ \\
& dc. process & 0.184 & 3 & 4628 & $<0.01$ \\
Varicose veins & dc. structure & 0.178 & 4 & 4627 & $<0.01$ \\
& dc. outcome & 0.063 & 3 & 4628 & $<0.01$ \\
& dc & 0.008 & 3 & 707 & $<0.05$ \\
Rheumatoid arthritis & dc. process & 0.187 & 3 & 704 & $<0.01$ \\
& dc. structure & 0.081 & 4 & 703 & $<0.01$ \\
& dc. outcome & 0.095 & 3 & 704 & $<0.01$ \\
& dc & 0.041 & 3 & 2753 & $<0.01$ \\
Spinal disc herniation & dc. process & 0.164 & 3 & 2750 & $<0.01$ \\
& dc. structure & 0.139 & 4 & 2749 & $<0.01$ \\
& dc. outcome & 0.053 & 3 & 2750 & $<0.01$ \\
& dc & 0.052 & 3 & 611 & $<0.01$ \\
& dc. process & 0.233 & 3 & 608 & $<0.01$ \\
dc, demographic characteristics; outcome, experiences regarding outcome (number of improved elements, number of elements with no change
\end{tabular}

